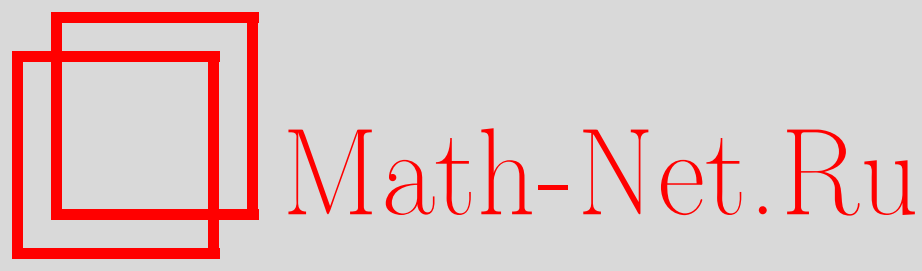

М. Ю. Ковалевский, В. Т. Мацкевич, А. Я. Разумный, Универсальность релаксационной структуры уравнений динамики сплошных сред и диссипативные скобки Пуассона, ТMФ, 2009, том 158, номер 2, 277-291

DOI: https://doi.org/10.4213/tmf6314

Использование Общероссийского математического портала Math-Net.Ru подразумевает, что вы прочитали и согласны с пользовательским соглашением http://www.mathnet.ru/rus/agreement

Параметры загрузки:

IP : 3.80 .253 .173

26 апреля 2023 г., 14:05:00

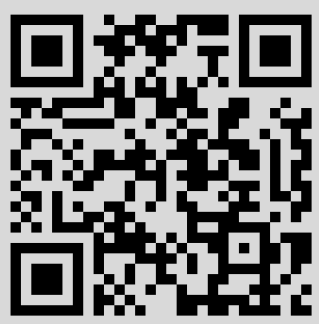




\title{
УНИВЕРСАЛЬНОСТЬ РЕЛАКСАЦИОННОЙ СТРУКТУРЫ УРАВНЕНИЙ ДИНАМИКИ СПЛОШНЫХ СРЕД И ДИССИПАТИВНЫЕ СКОБКИ ПУАССОНА
}

\begin{abstract}
Дано обобщение уравнений Гамильтона на динамические процессы с релаксацией. Диссипативные скобки Пуассона введены в терминах диссипативной функции. Получена универсальная структура релаксационных слагаемых в уравнениях динамики конденсированных сред. Этот результат проверен для бесструктурной жидкости, упругого твердого тела и квантовой жидкости. В рассмотренных примерах конденсированных сред получены выражения диссипативных скобок Пуассона для всего набора динамических параметров.
\end{abstract}

Ключевые слова: гамильтонов подход, энтропия, диссипативные скобки Пуассона, кинетические коэффициенты, твердое тело, квантовая жидкость, диссипативная функция.

\section{1. ВАРИАЦИОННЫЙ ПРИНЦИП И УРАВНЕНИЯ ГАМИЛЬТОНА}

В исследовании динамики многочастичных состояний важно учитывать релаксационные явления. Эта задача решается как методами статистической механики [1]-[5], так и в рамках феноменологических подходов [6]-[11]. В микроскопическом приближении использование представления сокращенного описания неравновесных процессов позволяет найти "огрубленный” статистический оператор в теории возмущений по какому-либо малому параметру (плотность, межчастичное взаимодействие, пространственные неоднородности). Динамические уравнения, получаемые в главном приближении теории возмущений, описывают адиабатические процессы. Учет следующего приближения приводит к возникновению интегралов столкновения или кинетических коэффициентов, которые описывают релаксацию на кинетическом или гидродинамическом этапе эволюции. Современное состояние этого раздела теоретической физики описано в монографиях [12], [13].

*Национальный научный центр "Харьковский физико-технический институт", г. Харьков, Украина. E-mail: mikov@kharkov.ua

${ }^{\dagger}$ Белгородский государственный университет, г. Белгород, Россия

${ }^{\ddagger}$ Харьковский национальный университет им. В. Н. Каразина, г. Харьков, Украина 
Гамильтонов формализм является эффективным феноменологическим методом исследования динамики разнообразных физических систем. Такой подход создавался для нормальных жидкостей, жидких кристаллов и твердых тел [6], [14], [15]. Он применялся к квантовым системам: сверхтекучим жидкостям, магнетикам, квантовым кристаллам [16]-[21], а также использовался для описания динамических процессов в физике плазмы [22] и кинетики взаимодействия спиновых волн [23].

Основополагающую роль в гамильтоновом подходе играет определение скобок Пуассона для всего набора динамических переменных. Обратимые динамические уравнения получаются исходя из вариационного принципа, а скобки Пуассона находятся на основе использования преобразований, оставляющих инвариантной кинематическую часть действия [21]. Учет диссипативных процессов в рамках феноменологических методов построения динамических уравнений осуществляется с использованием диссипативной функции и общих принципов термодинамики. Включение релаксационных процессов в рамках гамильтонова формализма проводилось в работах [10], [24]-[27]. В работе [24] рассмотрен кинетический этап эволюции. Введенные диссипативные скобки Пуассона приводят к релаксационному уравнению Ленарда-Балеску. Аналогичный подход [25] описывает релаксацию трехволновых динамических процессов. Релаксационная кинетика полимерных жидких кристаллов исследована в работе [10], диссипативная гидродинамика классической жидкости сформулирована в рамках гамильтонова подхода в работах [10], [26], [27]. Авторы этих работ ввели диссипативные скобки и получили необратимые уравнения динамики в квазигамильтоновой форме. Структура релаксационных слагаемых в этих динамических уравнениях не следует из вариационного принципа, а получается на основе применения уравнений Фоккера-Планка или других необратимых уравнений динамики [28].

Обратим внимание на существенное различие в построении динамических уравнений в микроскопическом и феноменологическом гамильтоновом подходе. Знание принципов статистической механики позволяет получить в рамках теории возмущений релаксационные уравнения. В гамильтоновом формализме динамические уравнения получаются без использования теории возмущений по малому параметру. Вопрос вывода общего вида релаксационных слагаемых в динамических уравнениях без использования методов теории возмущений в гамильтоновой механике остается открытым.

В настоящей работе построен гамильтонов формализм с учетом релаксационных процессов, как для нормальной жидкости, так и для конденсированных сред с нарушенной симметрией. Наряду со стандартным определением реактивной скобки Пуассона мы ввели диссипативные скобки Пуассона в терминах диссипативной функции. В силу принципа симметрии кинетических коэффициентов Онзагера диссипативная скобка Пуассона, в отличие от реактивной, является симметричной при перестановке входящих в нее динамических величин. Выяснено условие совпадения трансформационных свойств реактивных и диссипативных скобок Пуассона при конечных канонических преобразованиях. Показано, что структура релаксационных потоков нормальной жидкости приобретает универсальный вид, если диссипативная функция является однородным функционалом второго порядка. Сформулировано 
общее утверждение об универсальной структуре релаксационных слагаемых в динамических уравнениях и установлена его справедливость для двух рассмотренных в статье примеров конденсированных сред: сверхтекучей жидкости Не-ІІ и твердого тела. Формализм, используемый для введения релаксации в гамильтонов подход, позволяет установить более тесную связь с имеющимися статистическими методами исследования сплошных сред.

В соответствии с подходом механики сплошных сред лагранжиан произвольной физической системы имеет вид

$$
L=L_{\mathrm{k}}(\varphi, \dot{\varphi})-H(\varphi)
$$

где

$$
L_{\mathrm{k}}(\varphi, \dot{\varphi})=\int d^{3} x F_{\alpha}\left(x, \varphi\left(x^{\prime}\right)\right) \dot{\varphi}_{\alpha}(x)
$$

- кинематическая часть лагранжиана,

$$
H(\varphi)=\int d^{3} x \varepsilon(x, \varphi)
$$

- гамильтониан системы. Плотность энергии среды $\varepsilon\left(x, \varphi\left(x^{\prime}\right)\right)$, а также величи́ны $F_{\alpha}\left(x, \varphi\left(x^{\prime}\right)\right)$ являются функционалами от динамических переменных $\varphi_{\alpha}(x)$. Используя вариационную процедуру, получаем уравнения динамики для этих величин [21]:

$$
\dot{\varphi}_{\alpha}(x)=\int d^{3} x^{\prime} J_{\alpha \beta}^{-1}\left(x, x^{\prime}, \varphi\right) \frac{\delta H(\varphi)}{\delta \varphi_{\beta}\left(x^{\prime}\right)} \equiv\left\{\varphi_{\alpha}(x), H\right\}_{\mathrm{R}},
$$

где матрица $J_{\alpha \beta}\left(x, x^{\prime} ; \varphi\right)$ определяется равенством

$$
J_{\alpha \beta}\left(x, x^{\prime} ; \varphi\right)=\frac{\delta F_{\beta}\left(x^{\prime}, \varphi\right)}{\delta \varphi_{\alpha}(x)}-\frac{\delta F_{\alpha}(x, \varphi)}{\delta \varphi_{\beta}\left(x^{\prime}\right)} .
$$

Она связана со скобками Пуассона величин $\varphi_{\alpha}(x)$ соотношением

$$
\left\{\varphi_{\alpha}(x), \varphi_{\beta}\left(x^{\prime}\right)\right\}_{\mathrm{R}} \equiv J_{\alpha \beta}^{-1}\left(x, x^{\prime} ; \varphi\right) .
$$

Для различия обычной (реактивной) скобки Пуассона и диссипативной скобки реактивную скобку мы снабжаем индексом R. Из равенства (1.2) видно, что скобки (1.3) антисимметричны относительно перестановки $\alpha \leftrightarrow \beta, x \leftrightarrow x^{\prime}$ и удовлетворяют тождествам Лейбница и Якоби:

$$
\begin{aligned}
\left\{\varphi_{\alpha}(x), \varphi_{\beta}\left(x^{\prime}\right)\right\}_{\mathrm{R}}= & -\left\{\varphi_{\beta}\left(x^{\prime}\right), \varphi_{\alpha}(x)\right\}_{\mathrm{R}}, \\
\left\{\varphi_{\alpha}(x) \varphi_{\beta}\left(x^{\prime}\right), \varphi_{\gamma}\left(x^{\prime \prime}\right)\right\}_{\mathrm{R}}= & \varphi_{\alpha}(x)\left\{\varphi_{\beta}\left(x^{\prime}\right), \varphi_{\gamma}\left(x^{\prime \prime}\right)\right\}_{\mathrm{R}}+ \\
& +\varphi_{\beta}\left(x^{\prime}\right)\left\{\varphi_{\alpha}(x), \varphi_{\gamma}\left(x^{\prime \prime}\right)\right\}_{\mathrm{R}}, \\
\left\{\varphi_{\alpha}(x),\left\{\varphi_{\beta}\left(x^{\prime}\right), \varphi_{\gamma}\left(x^{\prime \prime}\right)\right\}_{\mathrm{R}}\right\}_{\mathrm{R}} & +\left\{\varphi_{\beta}\left(x^{\prime}\right),\left\{\varphi_{\gamma}\left(x^{\prime \prime}\right), \varphi_{\alpha}(x)\right\}_{\mathrm{R}}\right\}_{\mathrm{R}}+ \\
& +\left\{\varphi_{\gamma}\left(x^{\prime \prime}\right),\left\{\varphi_{\alpha}(x), \varphi_{\beta}\left(x^{\prime}\right)\right\}_{\mathrm{R}}\right\}_{\mathrm{R}}=0 .
\end{aligned}
$$

Для произвольных функционалов $A(\varphi), B(\varphi)$ скобки Пуассона определяем равенством

$$
\{A, B\}_{\mathrm{R}} \equiv \int d^{3} x \int d^{3} x^{\prime} \frac{\delta A}{\delta \varphi_{\alpha}(x)} J_{\alpha \beta}^{-1}\left(x, x^{\prime} ; \varphi\right) \frac{\delta B}{\delta \varphi_{\beta}\left(x^{\prime}\right)} .
$$


Функциональное выражение плотности энергии $\varepsilon\left(x, \varphi\left(x^{\prime}\right)\right)$ позволяет описывать динамику сплошных сред с произвольным характером пространственных неоднородностей. При этом возникают функциональные уравнения движения, которые очень сложны для анализа. Исследование динамических уравнений значительно упрощается в длинноволновом пределе, когда характерные размеры пространственных неоднородностей динамических переменных $L$ много больше длины свободного пробега $l$ (гидродинамическое приближение $L \gg l$ ). В этом случае выражение для плотности энергии становится локальной функцией набора параметров сокращенного описания $\varepsilon\left(x, \varphi\left(x^{\prime}\right)\right) \approx \varepsilon(\varphi(x), \nabla \varphi(x))$, и функциональные уравнения динамики переходят в дифференциальные уравнения.

Конечные преобразования

$$
\varphi_{\alpha}(x) \rightarrow \varphi_{\alpha}^{\prime}(x)=\varphi_{\alpha}\left(x, \varphi\left(x^{\prime}\right)\right)
$$

оставляющие инвариантной кинематическую часть действия

$$
L_{\mathrm{k}}(\varphi, \dot{\varphi})=\int d^{3} x F_{\alpha}(x ; \varphi) \dot{\varphi}_{\alpha}(x)=L_{\mathrm{k}}\left(\varphi^{\prime}, \dot{\varphi}^{\prime}\right)=\int d^{3} x F_{\alpha}\left(x ; \varphi^{\prime}\right) \dot{\varphi}_{\alpha}^{\prime}(x),
$$

являются каноническими, если выполняется равенство

$$
F_{\beta}\left(x^{\prime} ; \varphi\right)=\int d^{3} x F_{\alpha}\left(x ; \varphi^{\prime}\right) \frac{\delta \varphi_{\alpha}^{\prime}(x)}{\delta \varphi_{\beta}\left(x^{\prime}\right)}
$$

С учетом формул $(1.2),(1.7)$ представим условие каноничности в виде

$$
J_{\alpha \beta}\left(x, x^{\prime} ; \varphi\right)=\int d^{3} x_{1} d^{3} x_{2} \frac{\delta \varphi_{\lambda}^{\prime}\left(x_{1}\right)}{\delta \varphi_{\alpha}(x)} J_{\lambda \nu}\left(x_{1}, x_{2} ; \varphi^{\prime}\right) \frac{\delta \varphi_{\nu}^{\prime}\left(x_{2}\right)}{\delta \varphi_{\beta}\left(x^{\prime}\right)},
$$

откуда получаем условие каноничности преобразования для обратной матрицы

$$
J_{\beta \gamma}^{-1}\left(x^{\prime}, x^{\prime \prime} ; \varphi\right)=\int d^{3} y_{1} d^{3} y_{2} \frac{\delta \varphi_{\beta}\left(x^{\prime}\right)}{\delta \varphi_{\delta}^{\prime}\left(y_{1}\right)} J_{\delta \rho}^{-1}\left(y_{1}, y_{2} ; \varphi^{\prime}\right) \frac{\delta \varphi_{\gamma}\left(x^{\prime \prime}\right)}{\delta \varphi_{\rho}^{\prime}\left(y_{2}\right)} .
$$

Это соотношение приводит к инвариантности скобок Пуассона при канонических преобразованиях (1.6)

$$
\{A, B\}_{\mathrm{R}}=\int d^{3} x \int d^{3} x^{\prime} \frac{\delta A}{\delta \varphi_{\alpha}^{\prime}(x)} J_{\alpha \beta}^{-1}\left(x, x^{\prime} ; \varphi^{\prime}\right) \frac{\delta B}{\delta \varphi_{\beta}^{\prime}\left(x^{\prime}\right)} .
$$

Убедимся в ковариантности уравнений динамики при канонических преобразованиях (1.6). Действительно, в силу (1.9) нетрудно видеть, что из (1.1) следует справедливость уравнения динамики

$$
\dot{\varphi}_{\alpha}^{\prime}(x)=\int d^{3} x^{\prime} J_{\alpha \beta}^{-1}\left(x, x^{\prime} ; \varphi^{\prime}\right) \frac{\delta H^{\prime}\left(\varphi^{\prime}\right)}{\delta \varphi_{\beta}^{\prime}\left(x^{\prime}\right)},
$$

где $H^{\prime}\left(\varphi^{\prime}\right) \equiv H\left(\varphi\left(\varphi^{\prime}\right)\right)$. 
При построении динамических уравнений механики сплошных сред важную роль играют законы сохранения, тесно связанные со свойствами симметрии гамильтониана. Набор аддитивных интегралов движения немагнитной среды состоит из гамильтониана, импульса и массы

$$
\gamma_{a}=H, P_{k}, M \equiv \int d^{3} x \zeta_{a}(x), \quad a=0, k, 4, \quad k=1,2,3,
$$

а законы сохранения в дифференциальной форме имеют вид

$$
\dot{\zeta}_{a}(x)=-\nabla_{k} \zeta_{a k}(x) .
$$

Имеет место представление плотности потоков аддитивных интегралов движения в терминах скобок Пуассона от соответствующих плотностей $\zeta_{a}[29]$ :

$$
\begin{aligned}
\zeta_{a k}(x) & =-\delta_{a k} \varepsilon(x)+\int d^{3} x^{\prime} x_{k}^{\prime} \int_{0}^{1} d \lambda\left\{\zeta_{a}(y), \varepsilon\left(y^{\prime}\right)\right\}_{\mathrm{R}}, \quad a \neq 0, \\
\zeta_{0 k}(x) & =\frac{1}{2} \int d^{3} x^{\prime} x_{k}^{\prime} \int_{0}^{1} d \lambda\left\{\varepsilon(y), \varepsilon\left(y^{\prime}\right)\right\}_{\mathrm{R}}, \\
y & \equiv x+\lambda x^{\prime}, \quad y^{\prime} \equiv x-(1-\lambda) x^{\prime} .
\end{aligned}
$$

При выводе (1.11) учтены свойства симметрии гамильтониана:

$$
\begin{array}{ll}
\{M, \varepsilon(x)\}_{\mathrm{R}}=0, & M \equiv \int d^{3} x \rho(x), \\
\left\{P_{i}, \varepsilon(x)\right\}_{\mathrm{R}}=\nabla_{i} \varepsilon(x), & P_{i} \equiv \int d^{3} x \pi_{i}(x), \\
\left\{L_{i}, \varepsilon(x)\right\}_{\mathrm{R}}=\varepsilon_{i k l} x_{k} \nabla_{l} \varepsilon(x), & L_{i} \equiv \varepsilon_{i k l} \int d^{3} x x_{k} \pi_{l}(x),
\end{array}
$$

где $L_{i}$ - орбитальный момент. Первое соотношение отражает свойство фазовой инвариантности гамильтониана, второе - трансляционную инвариантность, последнее соотношение описывает инвариантность гамильтониана относительно поворотов в конфигурационном пространстве.

В процессе эволюции сплошной среды происходит ее деформирование. Описание деформации использует понятия лагранжевых и эйлеровых координат. Эйлеровы координаты $x_{k}=x_{k}(\xi, t)$ задают положение частицы среды в текущий момент времени и вектор смещения $u_{k}(x)$ связывает лагранжеву координату $\xi_{k}$ с эйлеровой координатой $x_{k}: x_{k} \equiv \xi_{k}+u_{k}(x)$. Тензор дисторсии определяем равенством

$$
b_{i k}(x)=\nabla_{k} \xi_{i}(x)=\delta_{i k}-\nabla_{k} u_{i}(x) .
$$

Известно, что ненулевые скобки Пуассона для плотности импульса, массы, энтропии и тензора дисторсии имеют вид [14], [21]

$$
\begin{gathered}
\left\{\pi_{i}(x), \sigma\left(x^{\prime}\right)\right\}_{\mathrm{R}}=-\sigma(x) \nabla_{i} \delta\left(x-x^{\prime}\right), \quad\left\{\pi_{i}(x), \rho\left(x^{\prime}\right)\right\}_{\mathrm{R}}=\rho(x) \nabla_{i}^{\prime} \delta\left(x-x^{\prime}\right), \\
\left\{\pi_{i}(x), \pi_{j}\left(x^{\prime}\right)\right\}_{\mathrm{R}}=\pi_{j}(x) \nabla_{i}^{\prime} \delta\left(x-x^{\prime}\right)-\pi_{i}\left(x^{\prime}\right) \nabla_{j} \delta\left(x-x^{\prime}\right), \\
\left\{b_{i j}(x), \pi_{k}\left(x^{\prime}\right)\right\}_{\mathrm{R}}=b_{i k}\left(x^{\prime}\right) \nabla_{j}^{\prime} \delta\left(x-x^{\prime}\right) .
\end{gathered}
$$


Скобки Пуассона (1.14) служат основой построения нелинейных уравнений динамики классических сплошных сред. Особенности динамического описания жидкостей, кристаллов и жидких кристаллов в рамках гамильтонова подхода проявляются в разной зависимости плотности энергии от тензора дисторсии. Для изучаемой конденсированной среды гамильтониан является функционалом набора гидродинамических параметров, к которым относятся плотность энтропии, плотность импульса и плотность массы:

$$
H=H_{0}\left(\pi_{i}(x), \rho(x)\right)+V\left(\sigma\left(x^{\prime}\right), \rho\left(x^{\prime}\right), b\left(x^{\prime}\right)\right) .
$$

Всюду далее мы полагаем гамильтониан системы галилеево-инвариантным, т.е. его зависимость от плотности импульса имеет вид

$$
\begin{aligned}
H_{0} & =\int d^{3} x \frac{\pi^{2}(x)}{2 \rho(x)} \\
V & =\int d^{3} x^{\prime} \Phi\left(\rho\left(x^{\prime}\right), \sigma\left(x^{\prime}\right), b_{i j}\left(x^{\prime}\right)\right)
\end{aligned}
$$

Плотности массы вещества и числа частиц связаны соотношением $\rho(x)=m n(x)$, где $m$ - масса частицы.

\section{2. ДИНАМИКА НОРМАЛЬНОЙ ЖИДКОСТИ. УРАВНЕНИЯ НАВЬЕ-СТОКСА. КИНЕТИЧЕСКИЕ КОЭФФИЦИЕНТЫ}

Для рассматриваемой среды определим дифференциал плотности энергии и введем необходимые термодинамические величины:

$$
d \varepsilon=\frac{\partial \varepsilon}{\partial \sigma} d \sigma+\frac{\partial \varepsilon}{\partial \pi_{i}} d \pi_{i}+\frac{\partial \varepsilon}{\partial n} d n \equiv T d \sigma+\nu_{k} d \pi_{k}+\mu d n
$$

где $\rho(x)=m n(x), m$ - масса частицы и $n(x)$ - число частиц. Здесь температура $T$, макроскопическая скорость $\nu_{k}$ и химический потенциал $\mu$ связаны с термодинамическими силами $Y_{a} \equiv Y_{0}, Y_{k}, Y_{4}$ равенствами $T \equiv Y_{0}^{-1}, \nu_{k} \equiv-Y_{k} / Y_{0}, \mu \equiv-Y_{4} / Y_{0}$. Зададим термодинамический потенциал

$$
\omega\left(Y_{a}\right) \equiv Y_{a} \zeta_{a}-\sigma
$$

и запишем второе начало термодинамики

$$
d \omega=\zeta_{a} d Y_{a}=\frac{\partial \omega(Y)}{\partial Y_{a}} d Y_{a}
$$

Представление плотности потоков в виде скобок Пуассона от плотности аддитивных интегралов движения (1.12) позволяет найти выражения плотности потоков аддитивных интегралов движения в терминах термодинамического потенциала. Учитывая выражения для этих потоков (1.11) и скобки Пуассона (1.14), получаем (cм. [4])

$$
\zeta_{a k}^{(0)}=-\frac{\partial}{\partial Y_{a}}\left(\frac{\omega Y_{k}}{Y_{0}}\right), \quad a=0, k, 4
$$


Соотношения (2.3) и (2.4) показывают, что плотности аддитивных интегралов движения и соответствующие им потоки представлены в терминах плотности термодинамического потенциала. Эти формулы приводят к уравнениям гидродинамики Эйлера и описывают течение жидкости в адиабатическом приближении. Полная энтропия $\Sigma \equiv \int d^{3} x \sigma(x)$ сохраняется, т.е. $\dot{\Sigma}=0$, а уравнение динамики для плотности энтропии имеет вид

$$
\dot{\sigma}=-\nabla_{k}\left(\sigma \nu_{k}\right)
$$

Формулы (1.14), (2.3), (2.4) позволяют установить набор скобок Пуассона для плотностей аддитивных интегралов движения $\zeta_{a}(x)$ :

$$
\left\{\zeta_{a}(x), \zeta_{b}\left(x^{\prime}\right)\right\}_{\mathrm{R}}=\left[\delta_{a i} \zeta_{b}(x)+\delta_{b i} \zeta_{a}\left(x^{\prime}\right)+\delta_{a 0} \zeta_{b i}^{(0)}(x)+\delta_{b 0} \zeta_{a i}^{(0)}\left(x^{\prime}\right)\right] \nabla_{l}^{\prime} \delta\left(x-x^{\prime}\right) .
$$

Таким образом, матрица $J_{a b}^{-1}\left(x, x^{\prime} ; \zeta\right)$ найдена в явном виде для бесструктурной сплошной среды. Подставляя последнюю формулу в (1.5), получаем скобку Пуассона для произвольных величин

$$
\begin{aligned}
\{A, B\}_{\mathrm{R}} \equiv & \int d^{3} x\left[\frac{\delta B}{\delta \zeta_{b}(x)} \nabla_{i}\left(\frac{\delta A}{\delta \pi_{i}(x)} \zeta_{b}(x)+\frac{\delta A}{\delta \varepsilon(x)} \zeta_{b i}(x)\right)-\right. \\
& \left.-\frac{\delta A}{\delta \zeta_{b}(x)} \nabla_{i}\left(\frac{\delta B}{\delta \pi_{i}(x)} \zeta_{b}(x)+\frac{\delta B}{\delta \varepsilon(x)} \zeta_{b i}(x)\right)\right] .
\end{aligned}
$$

Найдем релаксационные слагаемые для уравнений гидродинамики. В этом случае в правой части уравнений (1.10) возникают дополнительные слагаемые - диссипативные потоки $\zeta_{a k}^{(1)}(x)$ :

$$
\dot{\zeta}_{a}(x)=-\nabla_{k}\left(\zeta_{a k}^{(0)}(x)+\zeta_{a k}^{(1)}(x)\right)
$$

Эти потоки представляют собой линейные выражения от градиентов термодинамических сил:

$$
\zeta_{a k}^{(1)}(x)=I_{a k, b l}(x) \nabla_{l} Y_{b}(x),
$$

где $I_{a k, b l}$ - обобщенные кинетические коэффициенты, которые удовлетворяют принципу симметрии кинетических коэффициентов Онзагера

$$
I_{a k, b l}=I_{b l, a k}
$$

Для изотропного состояния равновесия, в котором макроскопическая скорость равна нулю, отличны от нуля только следующие величины:

$$
I_{0 k, 0 l}=T^{2} \delta_{k l} \kappa, \quad I_{i j, l k}=T \zeta \delta_{j l} \delta_{i k}+T \eta\left(\delta_{i l} \delta_{j k}+\delta_{l k} \delta_{i j}-\frac{2}{3} \delta_{l j} \delta_{i k}\right),
$$

где $\kappa$ - коэффициент теплопроводности, $\zeta, \eta$ - коэффициенты первой и второй вязкости. В галилеево-инвариантных средах остальные кинетические коэффициенты обращаются в нуль. Следствием уравнений $(2.6),(2.7)$ и соотношения (2.1) является необратимое уравнение динамики для плотности энтропии

$$
\dot{\sigma}=-\nabla_{k}\left(\nu_{k} \sigma+j_{\sigma k}^{(1)}\right)+I
$$


Здесь плотность диссипативного потока энтропии $j_{\sigma k}^{(1)}$ и производство энтропии $I$ в терминах диссипативной функции имеют вид

$$
j_{\sigma k}=\frac{\partial \sigma}{\partial \zeta_{a}} \frac{\partial r}{\partial \nabla_{k} \partial \sigma / \partial \zeta_{a}}, \quad I=\nabla_{k} \frac{\partial \sigma}{\partial \zeta_{a}} \frac{\partial r}{\partial \nabla_{k} \partial \sigma / \partial \zeta_{a}}=2 r,
$$

где

$$
R \equiv \frac{1}{2} \int d^{3} x \nabla_{k} Y_{a}(x) I_{a k, b l}(x) \nabla_{l} Y_{b}(x)=\int d^{3} x r(x)
$$

- диссипативная функция. Положительная определенность производства энтропии в изотропном состоянии обеспечивается положительностью кинетических коэффициентов $\kappa>0, \zeta>0, \eta>0$.

Преобразуем уравнения гидродинамики (2.6) к виду, отражающему универсальность структуры диссипативных слагаемых этих уравнений. Из определения (2.12) видно, что диссипативная функция является квадратичным функционалом от величин $\nabla_{k} Y$. Тогда согласно теореме Эйлера получаем равенство

$$
\left.\int d^{3} x Y_{a}(x) \frac{\delta R(Y)}{\delta Y_{a}(x)}\right|_{I=\mathrm{const}}=2 R(Y)
$$

В результате уравнения гидродинамики (2.6) принимают вид

$$
\dot{\zeta}_{a}(x)=-\nabla_{k} \zeta_{a k}^{(0)}(x)+\frac{\delta R}{\delta\left(\delta \Sigma / \delta \zeta_{a}(x)\right)},
$$

где

$$
\left.\frac{\delta R}{\delta Y_{a}(x)}\right|_{I=\text { const }}=\left.\frac{\partial r(x)}{\partial Y_{a}(x)}\right|_{I=\text { const }}-\nabla_{k} \frac{\partial r(x)}{\partial \nabla_{k} Y_{a}(x)}, \quad \frac{\delta \Sigma}{\delta \zeta_{a}(x)}=Y_{a}(x) .
$$

Второе слагаемое в (2.13) имеет универсальный вид и определяется в терминах диссипативной функции.

\section{3. РЕЛАКСАЦИОННЫЕ ПРОЦЕССЫ И ДИССИПАТИВНЫЕ СКОБКИ ПУАССОНА}

Введем диссипативные скобки Пуассона и придадим гамильтонов вид релаксационным слагаемым в уравнениях (2.13). Для этого определим диссипативные скобки Пуассона для бесструктурной жидкости равенством

$$
\{A, B\}_{\mathrm{D}} \equiv \int d^{3} x d^{3} x^{\prime} \frac{\delta A}{\delta \zeta_{a}(x)}\left\{\zeta_{a}(x), \zeta_{b}\left(x^{\prime}\right)\right\}_{\mathrm{D}} \frac{\delta B}{\delta \zeta_{b}\left(x^{\prime}\right)} .
$$

C помощью индекса D мы отличаем диссипативную скобку от обычной реактивной скобки Пуассона. Покажем, что диссипативные скобки Пуассона для макроскопических параметров $\zeta_{a}(x)$, характеризующих сплошную бесструктурную среду, имеют вид

$$
\left\{\zeta_{a}(x), \zeta_{b}\left(x^{\prime}\right)\right\}_{\mathrm{D}} \equiv-\frac{1}{T_{0}} \nabla_{k} \nabla_{l}^{\prime}\left(I_{a k, b l}(x) \delta\left(x-x^{\prime}\right)\right),
$$


где $T_{0}$ - некоторая постоянная, имеющая размерность температуры. В соответствии с определением (3.2) диссипативную скобку можно выразить в терминах диссипативной функции (2.12). Легко видеть, что

$$
\left.\frac{\delta^{2} R}{\delta Y_{a}(x) \delta Y_{b}\left(x^{\prime}\right)}\right|_{I=\mathrm{const}}=\nabla_{k} \nabla_{j}^{\prime}\left(\delta\left(x-x^{\prime}\right) I_{a k, b j}(x)\right)=-T_{0}\left\{\zeta_{a}(x), \zeta_{b}\left(x^{\prime}\right)\right\}_{\mathrm{D}} .
$$

В силу принципа симметрии кинетических коэффициентов Онзагера (2.8) скобка Пуассона (3.2) симметрична:

$$
\left\{\zeta_{a}(x), \zeta_{b}\left(x^{\prime}\right)\right\}_{\mathrm{D}}=\left\{\zeta_{b}\left(x^{\prime}\right), \zeta_{a}(x)\right\}_{\mathrm{D}}
$$

Кроме того, для этой скобки справедливо правило Лейбница

$$
\{A B, C\}_{\mathrm{D}}=A\{B, C\}_{\mathrm{D}}+B\{A, C\}_{\mathrm{D}} .
$$

Отметим, что в соответствии с формулами (1.14), (3.5) имеют место соотношения

$$
\left\{\zeta_{a}(x), \Sigma\right\}_{\mathrm{R}}=0, \quad\left\{\zeta_{a}(x), H\right\}_{\mathrm{D}}=0 .
$$

Величина, канонически сопряженная к плотности энтропии, не входит в гамильтониан и является циклической переменной (см. [21], откуда следует первое равенство в (3.6)). Второе соотношение в (3.6) очевидно в силу определения (3.3). Если ввести обобщенную скобку Пуассона с помощью равенства

$$
\{A, B\}_{\mathrm{G}} \equiv \int d^{3} x \int d^{3} x^{\prime} \frac{\delta A}{\delta \zeta_{a}(x)}\left[\left\{\zeta_{a}(x), \zeta_{b}\left(x^{\prime}\right)\right\}_{\mathrm{R}}+\left\{\zeta_{a}(x), \zeta_{b}\left(x^{\prime}\right)\right\}_{\mathrm{D}}\right] \frac{\delta B}{\delta \zeta_{b}\left(x^{\prime}\right)},
$$

то, учитывая (3.6), уравнения динамики для параметров сокращенного описания с учетом релаксационных процессов можно записать в виде

$$
\dot{\zeta}_{a}(x)=\left\{\zeta_{a}(x), H-T_{0} \Sigma\right\}_{\mathrm{G}} .
$$

Диссипативные скобки (3.3) аналогичны диссипативным скобкам, введенным в работах [10], [26], [27], они приводят к одним и тем же динамическим уравнениям для нормальной жидкости.

Рассмотрим теперь произвольную конденсированную среду, для которой набор параметров сокращенного описания представляет собой некоторое множество величин $\varphi_{\alpha}(x)$. В соответствии с $(2.12)$, (3.3) считаем, что диссипативная функция и диссипативные скобки Пуассона связаны следующими равенствами:

$$
\begin{gathered}
R \equiv-\frac{T_{0}}{2} \int d^{3} x d^{3} x^{\prime} \frac{\delta \Sigma}{\delta \varphi_{\alpha}(x)}\left\{\varphi_{\alpha}(x), \varphi_{\beta}\left(x^{\prime}\right)\right\}_{\mathrm{D}} \frac{\delta \Sigma}{\delta \varphi_{\beta}\left(x^{\prime}\right)}=\int d^{3} x r(x), \\
\left\{\varphi_{\alpha}(x), \varphi_{\beta}\left(x^{\prime}\right)\right\}_{\mathrm{D}} \equiv-\left.\frac{1}{T_{0}} \frac{\delta^{2} R}{\delta\left(\delta \Sigma / \delta \varphi_{\alpha}(x)\right) \delta\left(\delta \Sigma / \delta \varphi_{\beta}\left(x^{\prime}\right)\right)}\right|_{I=\mathrm{const}} \equiv R_{\alpha \beta}\left(x, x^{\prime} ; \varphi\right) .
\end{gathered}
$$

Уравнение движения для величины $\varphi_{\alpha}(x)$ в рамках гамильтонова формализма, учитывающего диссипативные процессы, приобретает вид

$$
\dot{\varphi}_{\alpha}(x) \equiv\left\{\varphi_{\alpha}(x), H\right\}_{\mathrm{R}}-T_{0}\left\{\varphi_{\alpha}(x), \Sigma\right\}_{\mathrm{D}}=L_{\varphi_{\alpha}}^{\mathrm{R}}(x)+L_{\varphi_{\alpha}}^{\mathrm{D}}(x),
$$


где первое слагаемое имеет обычную гамильтонову форму, соответствующую реактивной скобке Пуассона, а второе слагаемое учитывает вклад релаксационных процессов и представлено в виде диссипативной скобки Пуассона. Согласно (3.9), (3.10) получаем

$$
\begin{aligned}
L_{\varphi_{\alpha}}^{\mathrm{D}}(x) & =-T_{0} \int d^{3} x^{\prime} \frac{\delta \Sigma}{\delta \varphi_{\beta}\left(x^{\prime}\right)}\left\{\varphi_{\alpha}(x), \varphi_{\beta}\left(x^{\prime}\right)\right\}_{\mathrm{D}}= \\
& =\int d^{3} x^{\prime} \frac{\delta \Sigma}{\delta \varphi_{\beta}\left(x^{\prime}\right)} \frac{\delta^{2} R}{\delta\left(\delta \Sigma / \delta \varphi_{\alpha}(x)\right) \delta\left(\delta \Sigma / \delta \varphi_{\beta}\left(x^{\prime}\right)\right)} .
\end{aligned}
$$

Отсюда следует универсальная структура диссипативных слагаемых в уравнении для величин $\varphi_{\alpha}(x)$,

$$
L_{\varphi_{\alpha}}^{\mathrm{D}}=\frac{\delta R}{\delta\left(\delta \Sigma / \delta \varphi_{\alpha}(x)\right)},
$$

поскольку имеет место свойство однородности диссипативного функционала второй степени, т.е. соотношение

$$
\int d^{3} x \frac{\delta \Sigma}{\delta \varphi_{\beta}(x)} \frac{\delta R}{\delta\left(\delta \Sigma / \delta \varphi_{\beta}(x)\right)}=2 R .
$$

Далее мы рассмотрим две конденсированные среды (сверхтекучая жидкость Не-ІІ и упругое твердое тело) и покажем на этих примерах справедливость соотношений (3.12), (3.13) и равенств

$$
\left\{\varphi_{\alpha}(x), \Sigma\right\}_{\mathrm{R}}=0, \quad\left\{\varphi_{\alpha}(x), H\right\}_{\mathrm{D}}=0,
$$

так что уравнения релаксационной динамики для параметров сокращенного описания приобретут вид, аналогичный формуле (3.8):

$$
\dot{\varphi}_{\alpha}(x)=\left\{\varphi_{\alpha}(x), H-T_{0} \Sigma\right\}_{\mathrm{G}} .
$$

В силу определения (3.9) диссипативная скобка Пуассона инвариантна и уравнения динамики с учетом релаксационных процессов будут ковариантны при канонических преобразованиях (1.6), если диссипативная скобка (3.9) удовлетворяет соотношению

$$
R_{\beta \gamma}\left(x^{\prime}, x^{\prime \prime} ; \varphi\right)=\int d^{3} y_{1} d^{3} y_{2} \frac{\delta \varphi_{\beta}\left(x^{\prime}\right)}{\delta \varphi_{\delta}^{\prime}\left(y_{1}\right)} R_{\delta \rho}\left(y_{1}, y_{2} ; \varphi^{\prime}\right) \frac{\delta \varphi_{\gamma}\left(x^{\prime \prime}\right)}{\delta \varphi_{\rho}^{\prime}\left(y_{2}\right)} .
$$

\section{4. СВЕРХТЕКУЧАЯ ЖИДКОСТЬ НЕ-ІІ. РЕЛАКСАЦИОННЫЕ УРАВНЕНИЯ И ДИССИПАТИВНЫЕ СКОБКИ ПУАССОНА}

Рассматриваемая сверхтекучая жидкость на гидродинамическом этапе эволюции характеризуется дополнительной величиной - сверхтекучей фазой $\phi$, которая является канонически сопряженной величиной к плотности числа частиц. Можно показать, что скобки Пуассона для этой величины с плотностями импульса, массы, энтропии имеют вид [21]

$$
\begin{gathered}
\left\{\pi_{i}(x), \phi\left(x^{\prime}\right)\right\}_{\mathrm{R}}=-\delta\left(x-x^{\prime}\right) \nabla_{i} \phi(x), \\
\left\{\rho(x), \phi\left(x^{\prime}\right)\right\}_{\mathrm{R}}=\delta\left(x-x^{\prime}\right), \quad\left\{\sigma(x), \phi\left(x^{\prime}\right)\right\}_{\mathrm{R}}=0 .
\end{gathered}
$$


В силу фазовой инвариантности гамильтониан зависит только от градиента сверхтекучей фазы $H=H\left(\sigma\left(x^{\prime}\right), \pi_{i}\left(x^{\prime}\right), \rho\left(x^{\prime}\right), p_{k}\left(x^{\prime}\right)\right)$, где $p_{k}=\nabla_{k} \varphi-$ сверхтекучий импульс. Для термодинамического потенциала $\omega(Y, \vec{p})$, который по-прежнему определяется равенством (2.2), справедливо основное термодинамическое соотношение [13]

$$
d \omega=\varepsilon d Y_{0}+\pi_{k} d Y_{k}+n d Y_{4}+\left(Y_{0} j_{l}+Y_{l} n\right) d p_{l},
$$

где $j_{k}$ - плотность потока числа частиц. Используя формулы (1.12), (1.14), (4.1), можно выразить плотности потоков аддитивных интегралов движения в терминах термодинамического потенциала [13]:

$$
\zeta_{a k}^{(0)}=-\frac{\partial}{\partial Y_{a}} \frac{\omega Y_{k}}{Y_{0}}+\frac{\partial \omega}{\partial p_{k}} \frac{\partial p_{0}}{\partial Y_{a}}, \quad p_{0} \equiv \frac{Y_{4}+Y_{k} p_{k}}{Y_{0}} .
$$

С учетом диссипативных процессов уравнения для сверхтекучей жидкости можно представить в виде

$$
\dot{\zeta}_{a}=-\nabla_{k}\left(\zeta_{a k}^{(0)}+\zeta_{a k}^{(1)}\right), \quad \dot{\varphi}=p_{0}+L_{\varphi}^{(1)},
$$

где реактивные потоки $\zeta_{a k}^{(0)}$ и $p_{0}$ описывают динамику жидкости в адиабатическом приближении. Диссипативные потоки $\zeta_{a k}^{(1)}$ и $L_{\varphi}^{(1)}$ получены в микроскопическом рассмотрении [13]:

$$
\zeta_{a k}^{(1)}=\nabla_{l} Y_{b} I_{b l, a k}+\nabla_{i} \frac{\partial \omega}{\partial p_{i}} I_{a k}, \quad L_{\varphi}^{(1)}=\nabla_{l} Y_{b} I_{b l}+\nabla_{i} \frac{\partial \omega}{\partial p_{i}} \bar{I} .
$$

Обобщенные кинетические коэффициенты $I_{b l, a k}, I_{a k}$ и $\bar{I}$ описывают релаксационные процессы в изучаемой сверхтекучей жидкости. Они связаны с коэффициентами вязкости $\zeta_{1}, \zeta_{2}, \zeta_{3}, \eta$ и теплопроводности $\kappa$ равенствами

$$
\begin{aligned}
I_{0 k, 0 l} & =T^{2} \delta_{k l} \kappa, \quad I_{b l}=\delta_{b l} T \zeta_{1}, \quad \bar{I}=T \zeta_{3}, \\
I_{l j, i k} & =T \zeta_{2} \delta_{j l} \delta_{i k}+T \eta\left(\delta_{i l} \delta_{j k}+\delta_{l k} \delta_{i j}-\frac{2}{3} \delta_{l j} \delta_{i k}\right) .
\end{aligned}
$$

Следствием уравнений (4.3), (4.4) является необратимое уравнение динамики для плотности энтропии $(2.10)$, где

$$
I=\nabla_{k} Y_{a} \zeta_{a k}^{(1)}+\nabla_{k} \frac{\partial \omega}{\partial p_{k}} L_{\varphi}^{(1)} \geqslant 0, \quad j_{\sigma k}^{(1)}=Y_{a} \zeta_{a k}^{(1)}+\frac{\partial \omega}{\partial p_{k}} L_{\varphi}^{(1)}
$$

- производство и плотность диссипативного потока энтропии сверхтекучей жидкости Не-ІІ. Формулы (4.4), (4.6) приводят к явному виду плотности диссипативной функции сверхтекучей жидкости

$$
r=\frac{1}{2}\left(\nabla_{k} Y_{a} I_{a k, b l} \nabla_{l} Y_{b}+\nabla_{k} \frac{\partial \omega}{\partial p_{k}} \bar{I} \nabla_{l} \frac{\partial \omega}{\partial p_{l}}\right)+\nabla_{k} Y_{a} I_{a k} \nabla_{l} \frac{\partial \omega}{\partial p_{l}} .
$$

Положительность производства энтропии в выражении (4.6) для изотропного случая (при $\left.Y_{k}=p_{k}=0\right)$ обеспечивается неравенствами для кинетических коэффициентов $\kappa \geqslant 0, \zeta_{3} \geqslant 0, \eta \geqslant 0, \zeta_{1}^{2} \leqslant \zeta_{2} \zeta_{3}$. Учитывая, что

$$
\left.\frac{\partial \omega}{\partial p_{k}}\right|_{Y}=-\left.\frac{\partial \sigma}{\partial p_{k}}\right|_{\zeta}
$$


и используя формулу (4.7), получаем уравнения сверхтекучей гидродинамики

$$
\dot{\zeta}_{a}(x)=-\nabla_{k} \zeta_{a k}^{(0)}(x)+\frac{\delta R}{\delta\left(\delta \Sigma / \delta \zeta_{a}(x)\right)}, \quad \dot{\varphi}(x)=p_{0}(x)+\frac{\delta R}{\delta(\delta \Sigma / \delta \varphi(x))},
$$

имеющие универсальный вид релаксационных слагаемых (см. (3.9)). Из явного вида диссипативной функции (4.7) также легко убедиться в справедливости соотношения (3.13) для рассматриваемой системы. Знание диссипативной функции (4.7) и использование формулы (3.9) позволяют найти диссипативные скобки Пуассона изучаемой сверхтекучей жидкости:

$$
\begin{gathered}
\left\{\zeta_{a}(x), \zeta_{b}\left(x^{\prime}\right)\right\}_{\mathrm{D}}=-\frac{1}{T_{0}} \nabla_{k} \nabla_{j}^{\prime}\left(\delta\left(x-x^{\prime}\right) I_{a k, b j}(x)\right), \\
\left\{\zeta_{a}(x), \phi\left(x^{\prime}\right)\right\}_{\mathrm{D}}=\frac{1}{T_{0}} \nabla_{k}\left(\delta\left(x-x^{\prime}\right) I_{a k}(x)\right), \quad\left\{\phi(x), \phi\left(x^{\prime}\right)\right\}_{\mathrm{D}}=-\frac{1}{T_{0}}\left(\delta\left(x-x^{\prime}\right) \bar{I}(x)\right) .
\end{gathered}
$$

\section{5. РЕЛАКСАЦИОННАЯ ТЕОРИЯ УПРУГОСТИ. ДИССИПАТИВНЫЕ СКОБКИ ПУАССОНА}

Для построения релаксационной теории упругости введем в рассмотрение тензор деформаций и векторы обратной решетки в терминах тензора дисторсии. Поскольку мы везде используем эйлерово описание, эти дополнительные макроскопические параметры также вводятся в терминах эйлеровых переменных. Определим тензор деформаций Коши-Грина равенством

$$
u_{i j}(x) \equiv \frac{1}{2}\left(\delta_{i j}-b_{\lambda i}(x) b_{\lambda j}(x)\right)
$$

где свертка в правой части выполнена по лагранжеву индексу. Учитывая формулы (1.14), запишем единственную нетривиальную скобку Пуассона для этой величины:

$$
\begin{aligned}
\left\{u_{i j}(x), \pi_{k}\left(x^{\prime}\right)\right\}_{\mathrm{R}} & =u_{i j k \lambda}(x) \nabla_{\lambda} \delta\left(x-x^{\prime}\right)-\delta\left(x-x^{\prime}\right) \nabla_{k} u_{i j}(x), \\
u_{i j k \lambda}(x) & \equiv\left(\frac{1}{2} \delta_{j k}-u_{j k}(x)\right) \delta_{i \lambda}+\left(\frac{1}{2} \delta_{i k}-u_{i k}(x)\right) \delta_{j \lambda} .
\end{aligned}
$$

Эйлеровы векторы обратной кристаллической решетки в деформированном состоянии определяем равенством

$$
b_{\alpha i}(x) \equiv \underline{b}_{\alpha j} b_{j i}(x),
$$

где $\underline{b}_{\alpha j}$ - лагранжев вектор обратной кристаллической решетки в недеформированном состоянии. Согласно (1.14), (5.3) нетривиальная скобка Пуассона для величин $b_{\alpha i}(x)$ с плотностью импульса имеет вид

$$
\left\{b_{\alpha i}(x), \pi_{k}\left(x^{\prime}\right)\right\}_{\mathrm{R}}=-\nabla_{i}\left(b_{\alpha k}(x) \delta\left(x-x^{\prime}\right)\right) .
$$

Плотность энергии твердого тела является функцией следующих параметров: $\varepsilon(x)=\varepsilon\left(\sigma(x), \pi_{i}(x), u_{k}(x), u_{i k}(x), b_{\alpha k}(x)\right)$. С учетом пространственной однородности плотности энергии (1.12) приходим к равенству $\partial \varepsilon(x) / \partial u_{k}(x)=0$. Поэтому плотность энергии и термодинамический потенциал не зависят от вектора смещения. 
Термодинамический потенциал является функцией термодинамических сил, векторов обратной решетки и тензора деформаций $\omega=\omega\left(Y_{a}, b_{\alpha k}, u_{i k}\right)$. Учитывая формулы (1.11) и скобки Пуассона (1.14), (5.2), (5.4), нетрудно показать, что плотности потоков аддитивных интегралов движения в рассматриваемом случае принимают вид

$$
\begin{gathered}
\zeta_{a k}^{(0)}=-\frac{\partial}{\partial Y_{a}} \frac{\omega Y_{k}}{Y_{0}}+\frac{\partial \omega}{\partial u_{l k}} \frac{\partial g_{l}}{\partial Y_{a}}+\frac{\partial \omega}{\partial b_{\alpha k}} \frac{\partial b_{0 \alpha}}{\partial Y_{a}}, \\
g_{l}=\frac{Y_{i}\left(2 u_{i l}-\delta_{i l}\right)}{Y_{0}}, \quad b_{0 \alpha}=\frac{Y_{i} b_{\alpha i}}{Y_{0}},
\end{gathered}
$$

где $a=0, k$. При выводе выражения (5.5) учтена теорема о связи плотности потоков аддитивных интегралов движения в состоянии локального равновесия [13]:

$$
Y_{a}\left(Y_{k} \zeta_{a}+Y_{0} \zeta_{a k}\right)=0
$$

С учетом скобок Пуассона (5.2), (5.4) для тензора деформаций и векторов решетки согласно (1.1) получаем уравнения динамики

$$
\dot{u}_{i k}=u_{i k \lambda l} \nabla_{\lambda} \nu_{l}-\nu_{l} \nabla_{l} u_{i k}, \quad \dot{b}_{\alpha i}=-\nabla_{i}\left(\nu_{k} b_{\alpha k}\right) .
$$

Эти уравнения совместно с (1.10) и выражением для потоков (5.5) и представляют собой уравнения упругости в адиабатическом приближении. Для плотности энтропии справедливо уравнение (2.5).

Учет релаксационных процессов осуществим, если добавить к реактивным потокам в правых частях уравнений (1.10), (5.6) слагаемые, соответствующие диссипативным потокам, т.е.

$$
\begin{aligned}
\dot{\zeta}_{a} & =-\nabla_{k}\left(\zeta_{a k}^{(0)}+\zeta_{a k}^{(1)}\right) \equiv L_{a}^{(1)}+L_{a}^{(2)}, \\
\dot{b}_{\alpha i} & =-\nabla_{i}\left(\left(\nu_{k}+\nu_{k}^{(1)}\right) b_{\alpha k}\right) \equiv L_{\alpha i}^{(1)}+L_{\alpha i}^{(2)}, \\
\dot{u}_{i k} & =u_{i k \lambda l} \nabla_{\lambda}\left(\nu_{l}+\nu_{l}^{(1)}\right)-\left(\nu_{l}+\nu_{l}^{(1)}\right) \nabla_{l} u_{i k} \equiv L_{i k}^{(1)}+L_{i k}^{(2)} .
\end{aligned}
$$

Используя соотношение (2.2) и уравнения (5.7), для плотности энтропии получаем уравнение движения (2.10), где

$$
\begin{aligned}
I & =\nabla_{k} Y_{a} \zeta_{a k}^{(1)}+D_{l} \nu_{l}^{(1)} \geqslant 0, \\
j_{\sigma k}^{(1)} & =Y_{a} \zeta_{a k}^{(1)}-\frac{\partial \omega}{\partial u_{k j}}\left(2 u_{j l}-\delta_{j l}\right) \nu_{l}^{(1)}-\frac{\partial \omega}{\partial b_{\alpha k}} b_{\alpha l} \nu_{l}^{(1)}, \\
D_{l} & \equiv-\frac{\partial \sigma}{\partial u_{i k}} \nabla_{l} u_{i k}+\left(2 u_{k l}-\delta_{k l}\right) \nabla_{i} \frac{\partial \sigma}{\partial u_{i k}}+2 \frac{\partial \sigma}{\partial u_{i k}} \nabla_{i} u_{l k}+b_{\alpha l} \nabla_{i} \frac{\partial \sigma}{\partial b_{\alpha i}} .
\end{aligned}
$$

Диссипативные потоки $\zeta_{a k}^{(1)}$ и $\nu_{l}^{(1)}$ являются линейными функциями градиентов термодинамических сил $\nabla_{k} Y_{a}$ и вектора $D_{l}$ :

$$
\zeta_{a k}^{(1)}=\nabla_{l} Y_{b} I_{b l, a k}+D_{l} I_{a k, l}, \quad \nu_{j}^{(1)}=\nabla_{l} Y_{b} I_{j, b l}+D_{l} \bar{I}_{j, l},
$$

5 Теоретическая и математическая физика, т. 158, № 2, 2009 г. 
а кинетические коэффициенты $I_{b l, a k}, I_{a k, l}, I_{l, a k}, \bar{I}_{j, l}$ удовлетворяют принципу симметрии Онзагера:

$$
I_{a k, b l}=I_{b l, a k}, \quad I_{a k, l}=I_{l, a k}, \quad \bar{I}_{j, l}=\bar{I}_{l, j} .
$$

В рассматриваемом случае плотность диссипативной функции имеет вид

$$
r=\frac{1}{2}\left(\nabla_{k} Y_{a} I_{a k, b l} \nabla_{l} Y_{b}+D_{k} \bar{I}_{k, l} D_{l}\right)+\nabla_{k} Y_{a} I_{a k, l} D_{l}
$$

Используя выражение для диссипативной функции (5.11), получаем универсальную структуру диссипативных потоков (см. (3.9)) в релаксационных уравнениях теории упругости:

$$
L_{a}^{(2)}(x)=\frac{\delta R}{\delta\left(\delta \Sigma / \delta \zeta_{a}(x)\right)}, \quad L_{\alpha i}^{(2)}(x)=\frac{\delta R}{\delta\left(\delta \Sigma / \delta b_{\alpha i}(x)\right)}, \quad L_{i k}^{(2)}(x)=\frac{\delta R}{\delta\left(\delta \Sigma / \delta u_{i k}(x)\right)}
$$

Для изотропного твердого тела, когда в состоянии равновесия $b_{\alpha k}=Y_{k}=0$, среда характеризуется пятью кинетическими коэффициентами. Три из них имеют вид (2.9), а два новых кинетических коэффициента определяются равенствами $\bar{I}_{i j} \equiv \delta_{i j} B, I_{a k, l} \equiv \delta_{a 0} \delta_{k l} A$. Кинетические коэффициенты вязкоупругости $A, B$ для положительности производства энтропии удовлетворяют неравенствам $B \geqslant 0$, $T^{2} \kappa B \geqslant A^{2}$. В заключение приведем диссипативные скобки Пуассона упругой среды. Учитывая определение (3.9) и явный вид плотности диссипативной функции (5.11), получаем

$$
\begin{aligned}
\left\{\zeta_{a}(x), \zeta_{b}\left(x^{\prime}\right)\right\}_{\mathrm{D}}= & -\frac{1}{T_{0}} \nabla_{k} \nabla_{j}^{\prime}\left(\delta\left(x-x^{\prime}\right) I_{a k, b j}(x)\right) \\
\left\{\zeta_{a}(x), \zeta_{\beta l}\left(x^{\prime}\right)\right\}_{\mathrm{D}}= & -\frac{1}{T_{0}} \nabla_{k} \nabla_{l}^{\prime}\left(\delta\left(x-x^{\prime}\right) I_{a k, j}(x) b_{\beta j}(x)\right) \\
\left\{b_{\alpha k}(x), b_{\beta l}\left(x^{\prime}\right)\right\}_{\mathrm{D}}= & -\frac{1}{T_{0}} \nabla_{k} \nabla_{l}^{\prime}\left(\delta\left(x-x^{\prime}\right) b_{\alpha i}(x) \bar{I}_{i, j}(x) b_{\beta j}(x)\right) \\
\left\{b_{\alpha k}(x), u_{i j}\left(x^{\prime}\right)\right\}_{\mathrm{D}}= & -\frac{1}{T_{0}} \nabla_{k}\left\{b _ { \alpha l } ( x ) \overline { I } _ { l , n } ( x ) \left[\delta\left(x-x^{\prime}\right) \nabla_{n} u_{i j}(x)+\right.\right. \\
& \left.\left.+\nabla_{p}\left(\delta\left(x-x^{\prime}\right) u_{i j n p}(x)\right)\right]\right\} \\
\left\{\zeta_{a}(x), u_{i j}\left(x^{\prime}\right)\right\}_{\mathrm{D}}= & -\frac{1}{T_{0}} \nabla_{k}\left\{I_{a k, l}(x)\left[\delta\left(x-x^{\prime}\right) \nabla_{l} u_{i j}(x)+\nabla_{p}\left(\delta\left(x-x^{\prime}\right) u_{i j l p}(x)\right)\right]\right\} \\
\left\{u_{i k}(x), u_{l j}\left(x^{\prime}\right)\right\}_{\mathrm{D}}= & -\frac{1}{T_{0}}\left\{\bar{I}_{n, m}(x)\left(\delta\left(x-x^{\prime}\right) \Gamma_{m, j l}(x)-u_{j l m q}(x) \nabla_{q} \delta\left(x-x^{\prime}\right)\right) \Gamma_{n, i k}(x)+\right. \\
& +\nabla_{p}\left[\overline { I } _ { n , m } ( x ) \left(\delta\left(x-x^{\prime}\right) \Gamma_{m, j l}(x)-\right.\right. \\
& \left.\left.\left.-u_{j l m q}(x) \nabla_{q} \delta\left(x-x^{\prime}\right)\right) u_{i k n p}(x)\right]\right\}
\end{aligned}
$$

где $\Gamma_{\lambda, l j} \equiv \nabla_{\lambda} u_{l j}+\nabla_{p} u_{l j \lambda p}-$ символ Кристоффеля первого рода. Как следует из вида этих диссипативных скобок Пуассона, справедливо равенство (3.14) и имеет место универсальность релаксационной структуры уравнений упругости в виде (3.15).

Благодарности. Работа выполнена при финансовой поддержке РФФИ (грант № 05-02-16663 (М. Ю. Ковалевский, В. Т. Мацкевич)). 


\section{Список литературы}

[1] Н. Н. Боголюбов, Проблемы динамической теории в статистической физике, Гостехиздат, М.-Л., 1946.

[2] Л. Каданов, Г. Бейм, Квантовая статистическая механика. Функиии Грина, равновесные и неравновесные процессы, Мир, М., 1964.

[3] Д. Н. Зубарев, Неравновесная статистическая термодинамика, Физматлит, М., 1971.

[4] А.И. Ахиезер, С.В. Пелетминский, Методь статистической физики, Наука, М., 1977.

[5] Р. Балеску, Равновесная и неравновесная статистическая механика, Т. 2, Мир, М., 1978.

[6] Г. Лэмб, Гидродинамика, Гостехиздат, М.-Л., 1947.

[7] Л. Д. Ландау, Е. М. Лифшиц, Теоретическая физика. Т. VI. Гидродинамика, Наука, М., 1986.

[8] К. Трусделл, Первоначальный курс рациональной механики сплошных сред, Мир, М., 1975.

[9] П. Жермен, Курс механики сплошных сред, Высшая школа, М., 1983.

[10] A. N. Beris, B. J. Edwards, Thermodynamics of Flowing Systems with Internal Microstructure, Oxford Engrg. Sci. Ser., 36, Oxford Univ. Press, New York, 1994.

[11] P. M. Chaikin, T. C. Lubensky, Principles of Condensed Matter Physics, Cambridge Univ. Press, Cambridge, 2000.

[12] Д. Н. Зубарев, В. Г. Морозов, Г. Репке, Статистическая механика неравновесных процессов, Т. 1, Физматлит, М., 2002.

[13] М. Ю. Ковалевский, С.В. Пелетминский, Статистическая механика квантовых жидкостей и кристаллов, Физматлит, М., 2006.

[14] I. E. Dzyaloshinskii, G. E. Volovick, Ann. Phys., 125:1 (1980), 67-97.

[15] В. В. Лебедев, Е. И. Кац, Динамика жидких кристаллов, УРСС, М., 1988.

[16] Л. Д. Ландау, ЖЭТФ, 11 (1941), 592.

[17] В. В. Лебедев, И. М. Халатников, ЖЭТФ, 75:6 (1978), 2312-2316.

[18] A. J. Leggett, Rev. Modern Phys., 47:2 (1975), 331-414.

[19] С.П. Новиков, И.А. Тайманов, Современные геометрические структуры и поля, МЦНМО, М., 2005.

[20] Д. В. Волков, А. А. Желтухин, Ю. П. Блиох, ФТТ, 13 (1971), 1668.

[21] А. А. Исаев, М. Ю. Ковалевский, С. В. Пелетминский, ЭЧАЯ, 27:2 (1996), 431-492.

[22] В. Е. Захаров, Е.А. Кузнецов, УФН, 167:11 (1997), 1137-1166.

[23] В. С. Львов, Нелинейные спиновые волны, Физматлит, М., 1987.

[24] A. N. Kaufman, Phys. Lett. A, 100:8 (1984), 419-422.

[25] P. J. Morrison, Phys. Lett. A, 100:8 (1984), 423-427.

[26] M. Grmela, Phys. Lett. A, 102:8 (1984), 355-358.

[27] M. Grmela, H. C. Öttinger, Phys. Rev. E, 56:6 (1997), 6620-6632; 6633-6655.

[28] V.L. Berdichevsky, Phys. Rev. E, 68:6 (2003), 066126.

[29] Ю. П. Вирченко, С. В. Пелетминский, Проблемы физической кинетики и физики твердого тела, ред. А. Г. Ситенко, Наукова думка, Киев, 1990, 63-77.

Поступила в редакцию 10.01.2008, после доработки 10.04.2008 